\title{
Science and Evidence: Separating Fact from Fiction
}

\author{
Dean R Hess PhD RRT FAARC
}

\author{
Introduction \\ What Is Evidence-Based Medicine? \\ Hierarchy of Evidence \\ Finding the Evidence \\ Asking the Question \\ Source Literature \\ Systematic Reviews \\ Meta-Analysis \\ Clinical Practice Guidelines \\ Therapy That Evidence Supports \\ Noninvasive Ventilation \\ Lung-Protective Ventilation \\ Ventilator Discontinuation Protocols \\ Therapy That Evidence Does Not Support \\ Weaning Parameters \\ Albuterol for ARDS \\ High Frequency Oscillatory Ventilation for Adults \\ Therapy With Equivocal Evidence \\ Airway Clearance \\ Aerosol Device \\ PEEP for ARDS
}

Why Isn't the Best Evidence Implemented Into Practice?

Summary

Evidence-based medicine (EBM) is the integration of individual clinical expertise with the best available research evidence from systematic research and the patient's values and expectations. A hierarchy of evidence can be used to assess the strength upon which clinical decisions are made. The efficient approach to finding the best evidence is to identify systematic reviews or evidence-based clinical practice guidelines. Respiratory therapies that evidence supports include noninvasive ventilation for appropriately selected patients, lung-protective ventilation, and ventilator discontinuation protocols. Evidence does not support use of weaning parameters, albuterol for ARDS, and high frequency oscillatory ventilation for adults. Therapy with equivocal evidence includes airway clearance, selection of an aerosol delivery device, and PEEP for ARDS. Although all tenets of EBM are not universally accepted, the principles of EBM nonetheless provide a valuable approach to respiratory care practice. Key Words: clinical practice guideline; evidence-base medicine; meta-analysis; systematic review. [Respir Care 2013; 58(10):1649-1661. (C) 2013 Daedalus Enterprises]

Dr Hess is affiliated with the Department of Respiratory Care, Massachusetts General Hospital, and with the Department of Anesthesia, Harvard Medical School, Boston Massachusetts, and is the Editor in Chief of RESPIRATORY CARE.

Dr Hess presented a version of this paper at the 28th New Horizons in Respiratory Care Symposium, "The Scientific Basis for Respiratory Care," at the AARC Congress 2012, held November 10-13, 2012, in New Orleans, Louisiana.
Dr Hess has disclosed relationships with Philips, ResMed, Breathe Technologies, Pari, Covidien, and Maquet.

Correspondence: Dean R Hess PhD RRT FAARC, Respiratory Care, Ellison 401, Massachusetts General Hospital, 55 Fruit Street, Boston MA 02114. E-mail: dhess@ partners.org.

DOI: $10.4187 /$ respcare. 02591 


\section{Introduction}

Early in my career, the proceedings of the Conference on the Scientific Basis of Respiratory Therapy, commonly called the Sugarloaf Conference, were published. ${ }^{1}$ These proceedings came as a shock to me and many other respiratory therapists at the time. The participants of this conference recognized that many accepted respiratory therapy practices were not supported by adequate investigation: that they were based on clinical impression rather than rigorously conducted studies. The publication of these proceedings brought the predominant respiratory therapy procedure at the time, intermittent positive-pressure breathing (IPPB), under considerable scrutiny. Because evidence for benefit of IPPB was not forthcoming, this therapy has become virtually extinct in the 40 years following that conference. Reading the proceedings of the Sugarloaf Conference was instrumental in sparking my interest in evidence-based practice.

The emergence of evidence-based medicine (EBM) as a systematic distinct entity is traced to $1992,{ }^{2}$ with publication of the Users' Guides to the Medical Literature. ${ }^{3}$ Today EBM has permeated all parts of healthcare practice. This paper is an extension of a paper I published from the 2003 New Horizons Symposium. ${ }^{4}$

Respiratory care practice demands evidence for the accuracy of diagnostic tests, and for the efficacy and safety of the treatments that we apply. The traditional sources of this evidence are lacking. Experts are inadequate, lectures are ineffective, and professional journals are overwhelming. Textbooks are a useful collection of large amounts of background information, but the information is often outdated soon after publication. Our clinical assessment skills increase over time, but our knowledge tends to decrease over time. We are often too busy with our professional and personal lives to read the increasing body of research that is published weekly. The principles of EBM provide the tools to incorporate the best evidence into everyday practice.

\section{What Is Evidence-Based Medicine?}

EBM is the integration of individual clinical expertise with the best available evidence from systematic research and the patient's values and expectations. 5 The best evidence is not static, but changes when better evidence becomes available. EBM does not devalue clinical skills and clinical judgment. To the contrary, EBM medicine demands a high level of clinical skill and judgment. The practice of EBM requires us to apply the evidence to the right patient, at the right time, in the right place, at the right dose, and using the right resources.

Clinical evidence comes from real clinical research among intact patients. Bench studies and animal studies do
Table 1. Hierarchy of Evidence

$\mathrm{N}$ of 1 randomized trial

Systematic reviews of randomized trials

Single randomized trial

Systematic reviews of observational studies addressing patientimportant outcomes

Single observational study addressing a patient-important outcome Physiologic studies

Unsystematic clinical observations

not trump patient studies. These lower levels of evidence can be supportive of human studies, but they should not be used to invalidate the results of clinical studies.

Patient values and expectations are an important part of EBM. For example, there is a compelling body of highlevel evidence supporting the use of noninvasive ventilation (NIV) in patients with an exacerbation of COPD. Some patients, however, may prefer not to receive NIV. Another example relates to the choice of an aerosol delivery device, where a patient may prefer to use a nebulizer rather than a metered-dose inhaler. Although this may contradict the bias of the clinician, the patient's choice should be respected.

EBM is not cookbook medicine or cost-cutting medicine. The best evidence needs to be extrapolated to the patient's unique pathophysiology and values. High-level evidence is used to treat the majority of patients (the typical patient), but there are there are cases where only lowlevel evidence is available. This requires wisdom to select the best evidence when caring for the individual patient. With the implementation of EBM, costs may increase, decrease, or remain unchanged.

\section{Hierarchy of Evidence}

A hierarchy of evidence can be used to assess the strength upon which clinical decisions are made (Table 1). ${ }^{3}$ Clinicians should use the highest available evidence when making clinical decisions. Note that evidence always exists, but may be weak. The strongest available evidence may be an unsystematic clinical observation or a generalization from physiologic studies. Regardless of the source, there is always evidence.

Randomization is an important attribute of higher levels of evidence. Randomized controlled trials are compared to observational studies in Figure 1. The highest level of evidence is an $\mathrm{N}$ of 1 randomized controlled trial (RCT). In the $\mathrm{N}$ of $1 \mathrm{RCT}$, patients undertake pairs of treatment periods in which they receive a target treatment in one period of each pair, and a placebo or alternative in the other. $^{6-16}$ The order of the target and control is randomized, and quantitative ratings are made for each treatment. The $\mathrm{N}$ of 1 RCT continues until both the patient and cli- 

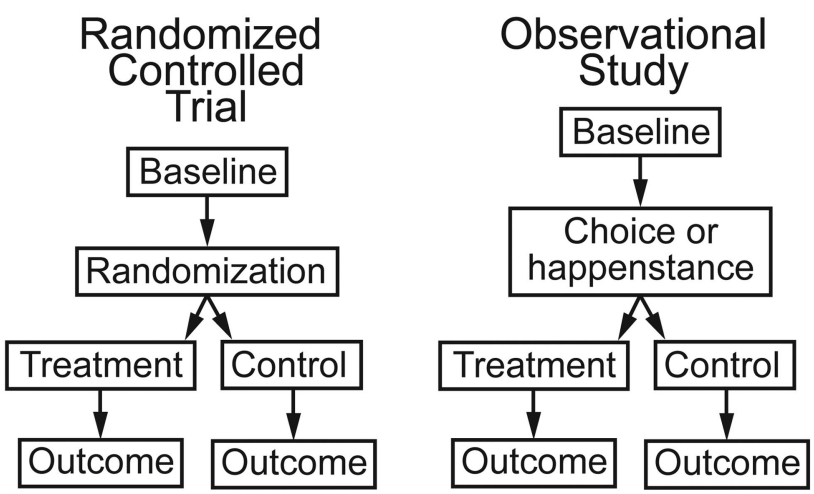

Fig. 1. Comparison between a randomized controlled study and an observational study. With the observational study, subjects are assigned to the treatment and control groups as a matter of choice or happenstance. With the randomized controlled study, subjects are randomly assigned to the treatment and control groups. As a result, there is less bias in the randomized study.

nician conclude that there is, or is not, benefit from the intervention.

There are some therapies for which there has not been a randomized trial, and for which one might argue that a randomized trial is either unethical or unnecessary. For example, it is unnecessary that a randomized trial be conducted to study the survival benefit of mechanical ventilation in patients with apnea, transfusion for massive blood loss, or antibiotics for bacterial pneumonia. In a tonguein-cheek paper arguing this point, Smith and Pell ${ }^{17}$ conducted a systematic review of RCTs to determine whether parachutes are effective in preventing major trauma related to gravitational challenge. They were unable to identify any RCTs of parachute intervention and concluded that, as with many interventions intended to prevent ill health, the effectiveness of parachutes has not been subjected to rigorous evaluation by using RCTs. They go on to suggest that everyone might benefit if the most radical protagonists of EBM organized and participated in a double blind, randomized, placebo controlled, crossover trial of the parachute.

In respiratory care the evidence to support many therapies is weak. Just because a therapy is unproven does not mean that it is wrong. But it also does not mean that it is right. And just because a therapy is new does not necessarily mean that it is better.

High-level studies are prospective, randomized, blinded, placebo-controlled, concealed allocation, parallel design, and assess patient-important outcomes (Table 2). Clinicians are often interested in physiologic outcomes, such as an improvement in arterial blood gas results. Patients, on the other hand, are more interested in outcomes such as survival. There are a number of examples in which an improvement in physiologic outcomes does not correlate with patient-important outcomes. High tidal volume $\left(\mathrm{V}_{\mathrm{T}}\right)$ in patients with ARDS improves the arterial blood gases, but mortality is lower for small $\mathrm{V}_{\mathrm{T}} \cdot{ }^{18}$ For patients with ARDS, inhaled nitric oxide improves $\mathrm{P}_{\mathrm{aO}_{2}}$, but not mortality. ${ }^{19}$ Mask CPAP for acute hypoxemic respiratory failure improves $\mathrm{P}_{\mathrm{aO}}$, but not intubation rate or mortality. ${ }^{20}$

Using the tools of EBM, metrics can be calculated such as event rate, relative risk, relative risk reduction, absolute risk reduction, number needed to treat (NNT), odds ratio (OR), and hazard ratio (Table 3). The confidence interval is defined as the range of values within which it is probable that the true value lies. The size of the confidence interval is determined by sample size and effect size. A larger sample size narrows the range of the confidence interval, increasing the precision of the study results and decreasing the risk of a false negative type 2 (beta) error (an effect exists but has not been detected). On the other hand, an excessively large study wastes resources, potentially places some patients at the risk of harm, increases the likelihood that a small and perhaps unimportant effect will be statistically significant, and increases the risk of a false positive type 1 (alpha) error (there appears to be an effect but there is none). The authors of high-level studies have conducted a power analysis during study design to determine the appropriate sample size.

\section{Finding the Evidence}

\section{Asking the Question}

Finding the best evidence begins with clearly articulating the question. PICO is a mnemonic used to help remember the key components of a well focused question: patient or problem, intervention, comparison, and outcomes.,21 Examples of PICO questions are shown in Table 4.

\section{Source Literature}

Primary studies supply the source evidence, but the information they contain needs critical assessment before application to clinical problems. Methods for conducting an online search to identify source literature are described in detail elsewhere. ${ }^{22-24} \mathrm{~A}$ commonly used search engine is Google (http://www.google.com). Conducting a search using Google is a quick way to find lots of information, but the amount of information obtained can be overwhelming and involve a considerable amount of time filtering through the search results. Much of the information that is found may be irrelevant. Moreover, the validity of information may be outdated or incorrect; it is almost never subjected to peer review. Google Scholar (http://scholar.google.com) provides searching of scholarly information, such as arti- 


\section{Science and Evidence: Separating Fact from Fiction}

Table 2. Characteristics of High-Level Studies

\begin{tabular}{ll}
\hline \hline \multicolumn{1}{c}{ Characteristic } & Description \\
\hline Prospective & $\begin{array}{c}\text { Prospective investigation of the factors that might cause a disorder in which a cohort of individuals } \\
\text { who do not have evidence of an outcome of interest but who are exposed to the putative cause } \\
\text { are compared with a concurrent cohort who are also free of the outcome but not exposed to the } \\
\text { putative cause. Both cohorts are then followed to compare the incidence of the outcome of } \\
\text { interest. } \\
\text { Allocation of individuals to groups by chance, usually done with the aid of table of random } \\
\text { numbers. This differs from systematic allocation (even and odd days of the month) or allocation } \\
\text { at the convenience or discretion of the investigator. } \\
\text { The participant of interest is unaware of whether subjects have been assigned to the experimental } \\
\text { or control group. Subjects, clinicians, those monitoring outcomes, assessors of outcomes, data } \\
\text { analysts, and those writing the paper can all be blinded. } \\
\text { Interventions without biologically active attributes. } \\
\text { Randomization is concealed if the person who is making the decision about enrolling a patient is } \\
\text { unaware of whether the next patient enrolled will be entered in the treatment or control group. } \\
\text { Wind (blinded or masked) } \\
\text { Placebo } \\
\text { Concealment } \\
\text { intervention is applied, and the outcome is identified for each subject. This is different than a } \\
\text { crossover study, in which subjects receive both the treatment and control intervention. }\end{array}$ \\
\hline
\end{tabular}

Table 3. Common Metrics Used in Evidence-Based Medicine

\begin{tabular}{|c|c|}
\hline Statistic & Description \\
\hline Event rate & $\begin{array}{l}\text { Proportion of subjects in a group in whom an event is observed. Control event rate and } \\
\text { experimental event rate refer to this in control and experimental groups of subjects, respectively. }\end{array}$ \\
\hline Relative risk & $\begin{array}{l}\text { Ratio of the risk of an event among an experimental group to the risk among the control group. A } \\
\text { relative risk }<1 \text { means benefit, a relative risk }>1 \text { means harm, and a relative risk }=1 \text { means } \\
\text { the intervention has no effect. }\end{array}$ \\
\hline Relative risk reduction & Estimate of the proportion of baseline risk that is removed by the therapy. \\
\hline Absolute risk reduction & $\begin{array}{l}\text { Difference in the absolute risk (percentage or proportion of subjects with an outcome) in the } \\
\text { exposed (experimental event rate) versus the unexposed (control event rate). }\end{array}$ \\
\hline Number needed to treat & Number of subjects who need to be treated to prevent one bad outcome. \\
\hline Odds ratio & $\begin{array}{l}\text { Ratio of the odds of an event in an exposed group to the odds of the same event in a group that is } \\
\text { not exposed. }\end{array}$ \\
\hline Hazard ratio & $\begin{array}{l}\text { Ratio of the hazard rates corresponding to the conditions described by } 2 \text { levels of an explanatory } \\
\text { variable; a measure of how often a particular event happens in one group compared to how often } \\
\text { it happens in another group, over time. }\end{array}$ \\
\hline
\end{tabular}

Table 4. Examples of PICO Questions

\begin{tabular}{|c|c|c|c|}
\hline Patient & Intervention & Comparison & Outcome \\
\hline $\begin{array}{l}\text { Adult with acute respiratory } \\
\text { distress syndrome }\end{array}$ & Low tidal volume & High tidal volume & Mortality \\
\hline Child with cystic fibrosis & Positive expiratory pressure & Conventional chest physiotherapy & Decline in lung function \\
\hline$>70$ years of age with COPD & Rehabilitation & Standard care & Exacerbations \\
\hline Child with acute asthma & Heliox & Standard care & Admission from emergency department \\
\hline
\end{tabular}

cles, dissertations, books, abstracts, and full text from publishers. Google Scholar ranks material and links to other documents that cite an important item you have identified.

MEDLINE is the National Library of Medicine database of indexed journal citations and abstracts. PubMed (www.pubmed.gov), the online interface to MEDLINE, is easy to use but overwhelming because of the large number of articles: more than 20 million. To search PubMed, enter search terms in the query box. The Advanced search feature allows content to be limited, such as to a specific 
Table 5. A Comparison of Narrative and Systematic Reviews

\begin{tabular}{lll}
\hline \hline \multicolumn{1}{c}{ Characteristic } & \multicolumn{1}{c}{ Narrative Review } & \multicolumn{1}{c}{ Systematic Review } \\
\hline Topic or question & Broad overview without a specific question & Narrow scope, with a specific question to be answered \\
Literature sources & Limited sources, typically not specified & Wide variety of specifically named databases \\
Literature selection & Unspecified, potentially biased & Pre-determined specific criteria for selection of papers \\
Literature appraisal & Unspecified, variable, and potentially biased & Critical review using specific criteria \\
Literature synthesis & Qualitative summary & Quantitative summary \\
Recommendations & Opinion, potentially biased, not evidence-based & Evidence-based \\
& & \\
(From reference 25.) & & \\
\hline
\end{tabular}

journal, author, or publication dates. Search filters can be selected from the left of the search page and the Related Articles feature in PubMed uses a word-weighted algorithm to compare words from the Title and Abstract of each citation. A comprehensive PubMed search for purposes of identifying the best evidence is overwhelming. Few individuals will have the time to read all of the papers identified in a PubMed search, assess the validity of the evidence, and develop strategies to incorporate such into everyday practice.

CINAHL (Cumulative Index to Nursing and Allied Health Literature, www.cinahl.com) is a nursing and allied health database. EMBASE (www.embase.com) is a large European database that is similar to MEDLINE in scope and content, with strengths in drugs and allied health disciplines. Up to $70 \%$ of citations in EMBASE are not included in MEDLINE. Ovid (www.ovid.com) provides medical information services to individuals in medical schools, hospitals, and academic institutions. Ovid provides access to a large selection of databases, including MEDLINE, CINAHL, and the other bibliographic databases. Most journals, including RESPIRATORY CARE, provide searching of journal contents by keyword or author directly from their home pages.

Many clinicians find it useful to scan journal contents monthly for new articles relevant to their practice. This is easily accomplished by subscribing to the e-mail alerts provided free of charge by journals. Read by QxMD (http:// www.qxmd.com) is an application for mobile devices that provides a single place to screen papers and read full text. It allows the user to follow specific journals and collections. It is also useful to use a citation manager to organize full text articles. My favorite application to accomplish this is Papers (http://www.papersapp.com), but similar features are available from EndNote, Reference Manager, RefWorks, and others. Citation manager software allows full text to be imported, and also allows one to conduct the online search from within the program and download directly to the citation manager.

\section{Systematic Reviews}

Narrative state-of-the-art reviews, such as those published in RESPIRATORY CARE from Journal Conferences and the New Horizons Symposia, are popular among readers. However, they are often limited by incomplete searches of the literature, intentional or unintentional bias by the authors, and failure to account for the quality of individual publications. Narrative reviews typically fail to effectively deal with studies having conflicting results, as they are written by experts who rely on their own experience and expertise rather than on a critical assessment of all available evidence. ${ }^{25}$

A systematic review is a summary of the literature that uses explicit methods, is based on a thorough literature search, performs a critical appraisal of individual studies, and uses statistical techniques to combine valid studies (meta-analysis). ${ }^{25}$ In a systematic review the primary evidence is rigorously identified and appraised. Unlike the traditional narrative review, a systematic review uses explicit methods. A systematic review details the methods by which papers were identified in the literature, it uses predetermined criteria for selection of papers to be included in the review, and it critically assesses the evidence and bases the review on the strength of that evidence. Narrative and systematic reviews are compared in Table 5.

Several sources can be searched for systematic reviews. PubMed can be searched using "systematic reviews" as article type. Ovid can be searched using the specific databases "EBM Reviews." The Cochrane Database (www. cochrane.org) is a rich source of systematic reviews, including many related to respiratory care. Systematic reviews and clinical practice guidelines may be outdated and should be supplemented by recent RCTs published after the publication date of the review or guideline.

\section{Meta-Analysis}

Individual studies should be placed in the context of the totality of evidence. ${ }^{26}$ The following reasons have been 
proposed to shift the collective focus from single studies to the accumulating body of evidence.

- Calibrate confidence in the estimates based on the consistency with other studies. Replication is a key step in science.

- Avoid premature closure about the magnitude of the effect before the estimate has moderated through repeated and independent evaluation. The most favorable result in support of a specific association is more likely to appear earlier than the least favorable one.

- Calibrate confidence in the estimates based on the true magnitude of precision. When results are inconsistent, the relative precision around the estimate of effect from a single study will overestimate the precision that the study contributes to the accumulating evidence.

- Improve the usefulness of results to clinical decision makers. Studies often adopt design features that help yield seemingly definitive findings. These features include using composite end points, surrogate end points, unfair comparisons, and selecting patients at high risk of the outcome.

- Prevent premature dismissal of a potentially effective intervention owing to concerns about nonsignificant results.

Meta-analysis is a statistical analysis that combines the results of several independent studies. ${ }^{25}$ A meta-analysis of RCTs is a higher level of evidence than a single RCT. A meta-analysis uses statistical methods to combine the results of several studies into a single pooled metric. Since it is based on a literature review, the meta-analysis is observational rather than experimental in nature.

The person conducting the meta-analysis has limited control over the availability of studies or the information reported in the individual studies. The studies included in the meta-analysis should be comparable, but the degree of comparability is subjective and determined by the person conducting the meta-analysis. Included studies should be identified from a comprehensive review of the literature, and unpublished data should be included to reduce the risk of publication bias. Tests for heterogeneity can be reported in the results of a meta-analysis. A common test of heterogeneity is the $\mathrm{I}^{2}$, which describes the percentage of the variability in effect estimates that is due to underlying differences in effect rather than chance. An $\mathrm{I}^{2}<20 \%$ represents minimal heterogeneity, $20-50 \%$ raises concern, and $>50 \%$ represents substantial heterogeneity. Betweenstudy variability should be examined by looking for differences in patients, interventions, outcome measurement, and methodology. ${ }^{3}$

In a meta-analysis, if the odds ratio (OR), risk ratio, or relative risk exceeds 1 , the likelihood of the outcome is greater in the treatment group. On the other hand, if it is below 1, the outcome is less likely in the treatment group. If the value is close to 1 , the outcomes in the treatment and control groups are more similar. If the confidence interval overlaps 1, the results are not significantly different from one another. A wider confidence interval indicates a less precise treatment effect, which is often due to smaller sample size.

The results of a meta-analysis are displayed as a forest plot. ${ }^{25}$ The outcome is placed on the $\mathrm{x}$-axis and the vertical line at 1.0 represents the no-effect line. Confidence intervals that cross this vertical line indicate that the study groups have equal risk of the outcome of interest. The square boxes are the point estimates for each study, and often (but not always) are presented as different-sized boxes, which correspond to the weight given to the study. A larger box reflects a higher weight assigned to that study. The cumulative treatment effect is represented by the diamond symbol at the bottom of the diagram. The center of the diamond represents the point estimate of the combined result, and the width of the diamond represents the $95 \%$ confidence interval of the point estimate.

\section{Clinical Practice Guidelines}

Evidence-based clinical practice guidelines ask relevant questions, systematically search the literature using explicit methodology, grade the level of the evidence, make recommendations, and grade the recommendations based upon the strength of the evidence. ${ }^{27}$ The recommendations of the evidence-based guidelines are supported by evidence, and the level of evidence is unambiguous and defensible. Clinical practice guidelines are often accompanied by systematic reviews and meta-analysis. They are often supported by professional organizations, including the American Association for Respiratory Care. ${ }^{28}$

The GRADE (Grading of Recommendations Assessment, Development, and Evaluation) approach is increasingly used to grade the quality of supporting evidence and the strength of recommendations in healthcare. ${ }^{29-38}$ The GRADE system provides a detailed stepwise process that defines the quality of the available evidence in the development of recommendations (Table 6). The value of GRADE is not that it eliminates judgments or disagreements about evidence and recommendations, but rather that it makes them transparent.

The public should trust clinical $^{39}$ practice guidelines only if the recommendations accurately reflect the underlying evidence about benefits and harms to individual patients. ${ }^{39}$ This requires a rigorous process for assembling, evaluating, and summarizing the evidence. Often this is the result of a rigorous systematic review. A second requirement is the process used to decide the recommended strategies that best offer a favorable balance of harms and 
Table 6. Strength of the Recommendation and Grade of Quality of the Evidence

\begin{tabular}{|c|c|c|}
\hline \multicolumn{3}{|c|}{ Strength of the Recommendation } \\
\hline Level & Strength & Description \\
\hline 1 & Stronger & $\begin{array}{l}\text { Benefits clearly outweigh the risks and burdens (or vice versa) for nearly } \\
\text { all patients }\end{array}$ \\
\hline 2 & Weaker & Risks and benefits are more closely balanced or are more uncertain \\
\hline \multicolumn{3}{|c|}{ Quality of the Evidence } \\
\hline Grade & Quality & Description \\
\hline A & High & $\begin{array}{l}\text { Well performed randomized controlled trials or overwhelming evidence } \\
\text { of some other sort. Further research is very unlikely to change our } \\
\text { confidence in the estimate of the effect. }\end{array}$ \\
\hline $\mathrm{B}$ & Moderate & $\begin{array}{l}\text { Randomized controlled trials that are less consistent, have flaws, or are } \\
\text { indirect in some way to the issue being graded, or very strong } \\
\text { evidence of some other sort. Further research is likely to have an } \\
\text { important impact on our confidence in the estimate of effect and may } \\
\text { change the estimate. }\end{array}$ \\
\hline $\mathrm{C}$ & Low & $\begin{array}{l}\text { Observational evidence (from observational studies, case series, or } \\
\text { clinical experience), or evidence from controlled trials with serious } \\
\text { flaws. Further research is very likely to have an important impact on } \\
\text { our confidence in the estimate of effect and is likely to change the } \\
\text { estimate. }\end{array}$ \\
\hline $\mathrm{D}$ & Very low & Any estimate of effect is very uncertain. \\
\hline
\end{tabular}

(From references 28 and 34.)

benefits. The Institute of Medicine developed the Appraisal of Guidelines for Research and Evaluation (AGREE) system to set of standards for rating the quality of the process of guideline development.

\section{Therapy That Evidence Supports}

\section{Noninvasive Ventilation}

In a Cochrane review of 14 studies, Ram et $\mathrm{al}^{40}$ reported that NIV decreased mortality (relative risk 0.52, 95\% CI 0.35-0.76), the need for intubation (relative risk $0.41,95 \% \mathrm{CI} 0.33-0.53$ ), and treatment failure (relative risk $0.48,95 \%$ CI $0.37-0.63$ ). The NNT was 10 (95\% CI 7-20), derived from 622 subjects in 10 studies. In another Cochrane review of CPAP of NIV for cardiogenic pulmonary edema, Vital et al ${ }^{41}$ included 21 studies involving 1,071 subjects. Compared to standard medical care, NIV significantly reduced hospital mortality (relative risk $0.6,95 \%$ CI $0.45-0.84$ ) and endotracheal intubation (relative risk $0.53,95 \%$ CI $0.34-0.83$ ) with NNT of 13 and 8 , respectively.

Clinical practice guidelines were prepared by an 18member guidelines panel as an initiative of the Canadian Critical Care Trials Group/Canadian Critical Care Society Noninvasive Ventilation Guidelines Group. ${ }^{42}$ Guidelines were graded with the GRADE approach. The final review included 146 RCTs. Guidelines were prepared on COPD exacerbation, asthma exacerbation, cardiogenic pulmonary edema, acute lung injury, chest trauma, immunosuppression in conjunction with acute respiratory distress or failure, bronchoscopy in patients with hypoxemia, adjunct to early liberation from mechanical ventilation, prevention of acute respiratory failure after low-risk surgery, prevention of acute respiratory, failure after high-risk surgery, treatment of acute respiratory failure after surgery, interface, and preferred mode for NIV. The following key points summarize this clinical practice guideline:

- NIV should be the first option for ventilatory support for patients with either a severe exacerbation of COPD or cardiogenic pulmonary edema.

- CPAP delivered by mask appears to be just as effective as NIV for patients with cardiogenic pulmonary edema.

- Patients with acute respiratory distress or hypoxemia, either in the postoperative setting or in the presence of immunosuppression, can be considered for a trial of NIV.

- Patients with COPD can be considered for a trial of early extubation to NIV.

A comparative effectiveness review commissioned by the Agency for Healthcare Research and Quality evaluated the evidence for NIV versus other typical treatments for acute respiratory failure. ${ }^{43}$ The review includes 44 studies $(4,122$ subjects) that compared NIV to supportive care, 5 (405 subjects) compared NIV to invasive ventilation, 12 (1,520 subjects) compared NIV to CPAP, and $12(1,463$ 
subjects) evaluated NIV for weaning or in patients postextubation. Most studies were conducted in patients with acute respiratory failure due to cardiogenic pulmonary edema or exacerbations of COPD. Compared with supportive care, NIV reduced hospital mortality (OR 0.56, 95\% CI 0.44-0.72), intubation rates (OR 0.31, 95\% CI 0.23-0.41), and hospital-acquired pneumonia. Limited evidence shows similar treatment effects across different settings and the possibility of less benefit in trials designed to replicate usual clinical practice.

\section{Lung-Protective Ventilation}

In the seminal ARDS Network study, ${ }^{18} 861$ patients with ARDS were randomly assigned to mechanical ventilation with a $\mathrm{V}_{\mathrm{T}}$ of $12 \mathrm{~mL} / \mathrm{kg}$ or $6 \mathrm{~mL} / \mathrm{kg}$, based on ideal (predicted) body weight and not actual body weight. Mortality for the control group $\left(12 \mathrm{~mL} / \mathrm{kg} \mathrm{V}_{\mathrm{T}}\right)$ was $39.8 \%$, and mortality for the experimental group $\left(6 \mathrm{~mL} / \mathrm{kg} \mathrm{V}_{\mathrm{T}}\right)$ was $31 \%$. The relative risk of mortality was lower for the $6 \mathrm{~mL} / \mathrm{kg}$ group (0.79), with a relative risk reduction of 0.21 compared to the $12 \mathrm{~mL} / \mathrm{kg}$ group. There was an absolute risk reduction for mortality of $8.8 \%$, resulting in an NNT of 11 patients.

In a multicenter prospective cohort study, Needham et $\mathrm{al}^{44}$ also found that higher survival is associated with lung-protective ventilation. They enrolled 485 subjects for 6,240 eligible ventilator settings, of which $41 \%$ adhered to lung-protective ventilation. After adjusting for the duration of ventilation and other relevant covariates, each additional ventilator setting adherent to lung-protective ventilation was associated with a $3 \%$ decrease in the risk of mortality over 2 years (hazard ratio $0.97,95 \%$ CI $0.95-$ $0.99, P=.002)$. Compared with no adherence, the estimated absolute risk reduction in 2-year mortality for a patient with $50 \%$ adherence to lung-protective ventilation was $4.0 \%(P=.01)$, and with $100 \%$ adherence was $7.8 \%$ $(P=.01)$. Average $\mathrm{V}_{\mathrm{T}}$ showed an independent linear relation with 2-year survival, with an $18 \%$ relative increase in the risk of mortality for each $1 \mathrm{~mL} / \mathrm{kg}$ predicted body weight increase in average $\mathrm{V}_{\mathrm{T}}$ over the duration of mechanical ventilation.

A meta-analysis by Serpa Neto ${ }^{45}$ suggests that use of lower $\mathrm{V}_{\mathrm{T}}$ also benefits patients who do not have ARDS. Their meta-analysis included 20 articles (2,822 subjects). Their results show a decrease in lung injury development (risk ratio $0.33,95 \%$ CI $0.23-0.47$, NNT 11 ) and mortality (risk ratio $0.64,95 \%$ CI $0.46-0.89$, NNT 23) in patients receiving ventilation with lower $\mathrm{V}_{\mathrm{T}}$. The results of lung injury development were similar when stratified by the type of study (randomized vs nonrandomized), and were significant only in randomized trials for pulmonary infection and only in nonrandomized trials for mortality. The meta-analysis also showed, in protective ventilation groups, a lower incidence of pulmonary infection (risk ratio 0.45 , 95\% CI 0.22-0.92, NNT 26), lower hospital stay, higher $\mathrm{P}_{\mathrm{aCO}_{2}}$, lower $\mathrm{pH}$, and similar $\mathrm{P}_{\mathrm{aO}} / \mathrm{F}_{\mathrm{IO}_{2}}$.

\section{Ventilator Discontinuation Protocols}

More than 10 years ago, evidence-based guidelines for weaning and discontinuing ventilatory support were published. ${ }^{46}$ One of the recommendations in these guidelines was that discontinuation protocols designed for non-physician healthcare professionals should be developed and implemented. More recently, a Cochrane systematic review and meta-analysis addressed the use of weaning protocols for reducing duration of mechanical ventilation in critically ill adult patients. ${ }^{47}$ Eleven trials that included 1,971 patients were included. Compared with usual care, the geometric mean duration of mechanical ventilation in the weaning protocol group was reduced by $25 \%$ (95\% CI $9-39 \%, P=.006,10$ trials), the duration of weaning was reduced by $78 \%$ (95\% CI 31-93\%, $P=.009,6$ trials), and stay in the ICU by $10 \%$ (95\% CI $2-19 \%, P=.02,8$ trials). Available evidence supports that the use of ventilator discontinuation protocols improves patient outcomes.

\section{Therapy That Evidence Does Not Support}

\section{Weaning Parameters}

In the past, many potential predictors of successful or unsuccessful liberation from mechanical ventilation have been proposed. Meade et $\mathrm{al}^{48}$ identified 65 observational studies of weaning predictors. For spontaneous breathing trials (SBT) the power of a positive test result for both breathing frequency and rapid shallow breathing index (RSBI) were limited (highest likelihood ratio 2.23), while the power of a negative test result was substantial (likelihood ratio $0.09-0.23$ ). Summary data suggest a similar predictive power for breathing frequency and RSBI. In the prediction of successful extubation, a breathing frequency $<38$ breaths/min (sensitivity $88 \%$, specificity $47 \%$ ) and a RSBI $<105$ breaths/min/L (sensitivity 65-96\%, specificity $0-73 \%$ ) are the most promising. A breathing frequency $>38$ breaths $/ \mathrm{min}$ and a RSBI of $>100$ breaths $/ \mathrm{min} / \mathrm{L}$ reduce the probability of successful extubation. Judging by areas under the receiver operator curve for all variables, none of these variables demonstrate more than modest accuracy in predicting weaning outcome.

Tanios and colleagues ${ }^{49}$ conducted a multicenter randomized blinded controlled trial in 304 subjects to determine the effect of including RSBI in a weaning protocol. In one group the RSBI was measured but not used in the decision to wean, but in the other group RSBI was measured and used. Subjects passing the screen received a 2-hour SBT, and those who passed the SBT were eligible 


\section{SCIEnCE AND Evidence: Separating FACt FROM Fiction}

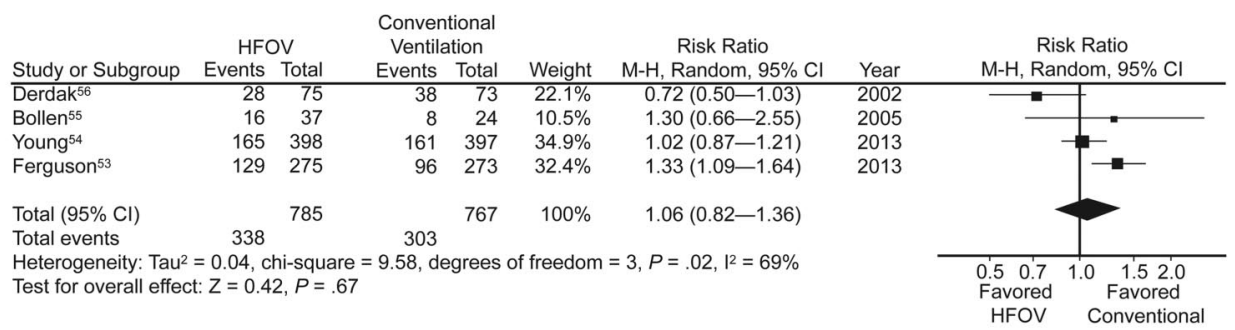

Fig. 2. A meta-analysis of mortality in 4 randomized controlled trials of high frequency oscillatory ventilation (HFOV) versus conventional ventilation. There is no difference in mortality, and a tendency toward lower mortality in the subjects receiving conventional ventilation. $\mathrm{M}-\mathrm{H}$ Mantel-Haenszel test. (Data from references 53-56.)

for extubation. The median duration for weaning time was significantly shorter in the group where RSBI was not used $(2.0 \mathrm{~d}$ vs $3.0 \mathrm{~d}, P=.04)$. There was no difference with regard to the extubation failure, in-hospital mortality rate, tracheostomy, or unplanned extubation. The results of this study suggest that use of RSBI might result in prolonged weaning time. These authors recommend that RSBI should not be used routinely in weaning decision-making.

A properly monitored SBT is very safe and effectively identifies patients ready for liberation from mechanical ventilation. ${ }^{46}$ Given that weaning parameters are not highly predictive of readiness for an SBT or readiness for extubation, weaning parameters can be omitted from practice, with emphasis placed on performance of an SBT. Common criteria for discontinuation of an SBT include tachypnea, desaturation, tachycardia or bradycardia, hypertension or hypotension, agitation, diaphoresis, or anxiety. These are identified at the bedside by a clinician, such as a respiratory therapist, without the need for weaning parameters.

\section{Albuterol for ARDS}

In the past it was believed that inhaled $\beta_{2}$ agonists were benign, and might help, in all mechanically ventilated patients. In many hospitals they are commonly administered to all mechanically ventilated patients. Moreover, there was some experimental and clinical evidence that $\beta_{2}$ agonists accelerate resolution of pulmonary edema. Thus, the ARDS Network conducted a multicenter randomized, placebo-controlled clinical trial in which 282 mechanically ventilated subjects with ARDS were randomized to receive aerosolized albuterol or placebo every 4 hours for up to 10 days. ${ }^{50}$ Ventilator-free days were not significantly different between the albuterol and placebo groups (means of $14.4 \mathrm{~d}$ and $16.6 \mathrm{~d}$, respectively). Mortality before hospital discharge was not significantly different between the albuterol and placebo groups $(23.0 \%$ and $17.7 \%$, respectively). In another multicenter placebo-controlled trial, ${ }^{51}$ 328 mechanically ventilated subjects with ARDS onset were randomly assigned to receive either intravenous sal- butamol or placebo for up to 7 days. Salbutamol increased 28 -day mortality (34\% vs $23 \%$, risk ratio $1.47,95 \% \mathrm{CI}$ 1.03-2.08). These data suggest that administration of $\beta_{2}$ agonists to mechanically ventilated patients with ARDS is unlikely to be beneficial, and could worsen outcomes. Routine use of $\beta_{2}$-agonist therapy in mechanically ventilated patients with ARDS cannot be recommended.

\section{High Frequency Oscillatory Ventilation for Adults}

High frequency oscillatory ventilation (HFOV) theoretically meets the goals of a strategy of lung-protective ventilation, with small $\mathrm{V}_{\mathrm{T}}$ lung recruitment. The use of HFOV in adults with ARDS has been controversial. ${ }^{52}$ Sud et al ${ }^{53}$ conducted a meta-analysis of 8 RCTs. In subjects randomized to HFOV, mortality was significantly reduced (risk ratio $0.77,95 \%$ CI $0.61-0.98, P=.03$ ), and treatment failure (refractory hypoxemia, hypercapnia, hypotension, or barotrauma) resulting in discontinuation of assigned therapy was less likely (risk ratio $0.67,95 \%$ CI $0.46-0.99$, $P=.04)$. There are several criticisms, however, of this meta-analysis. First, it combined studies of adults and children. Second, several of the studies were published in abstract only. Recently, 2 large RCTs of the use of HFOV in adults were published.54,55 One of these reported no difference between conventional ventilation and HFOV, ${ }^{55}$ but the other reported worse outcomes for HFOV. ${ }^{54}$ An updated meta-analysis of 4 randomized RCTs ${ }^{54-57}$ in adults, comparing HFOV and conventional ventilation shows no difference in mortality (Fig. 2), although the heterogeneity is large $\left(\mathrm{I}^{2}=69 \%\right)$. The accumulated evidence does not support the use of HFOV in adults with ARDS.

\section{Therapy With Equivocal Evidence}

\section{Airway Clearance}

Clearance of airway secretions is a primary therapy for patients with cystic fibrosis. Over the years, a variety of airway clearance therapies (ACTs) have been developed. The Cystic Fibrosis Foundation established a committee to 
examine the clinical evidence for each therapy and provide guidance for their use. ${ }^{58} \mathrm{~A}$ systematic review identified 7 unique reviews and 13 additional controlled trials that addressed 1 or more of the comparisons of interest and were deemed eligible for inclusion. Recommendations for use of the ACTs were made, balancing the quality of evidence and the potential harms and benefits. The committee determined that, although there is a paucity of controlled trials that assess the long-term effects of ACTs, the evidence of quality overall for their use in cystic fibrosis is fair and the benefit is moderate. The committee recommends that airway clearance should be performed on a regular basis in all patients with cystic fibrosis. However, no ACT was found to be superior to the others. Therefore, it was recommended that the prescription of ACTs should be individualized.

Ides and colleagues ${ }^{59}$ conducted a systematic review of ACT for patients with COPD. They found that studies that provide solid evidence of the effectiveness of different ACTs in patients with COPD are scarce. The available evidence indicates that active breathing techniques, such as active cycle of breathing techniques, autogenic drainage, and forced expiration, can be effective in the treatment of COPD. However, the evidence for passive techniques such as postural drainage and percussion is low. Other techniques, such as intrapulmonary percussive ventilation, positive expiratory pressure, and NIV, have little evidence because of the small number of studies. Little evidence was also found for the combined use of active techniques and techniques such as oscillating positive expiratory pressure, postural drainage, and vibration in COPD.

There is a dearth of high-level evidence related to ACTs. Studies in this field are hampered by small sample sizes, crossover designs, assessment of a single treatment session, less than careful attention to technique, surrogate outcome measures, lack of blinding, and statistical concerns. I have suggested the following hierarchy of questions that might be asked when considering ACT for a patient. ${ }^{10}$

- Is there a pathophysiologic rationale for use of the therapy? Is the patient experiencing difficulty in clearing of secretions? Are retained secretions affecting lung function in an important way, such as gas exchange or lung mechanics? Note that the production of large amounts of sputum does not necessarily mean that the patient is experiencing difficulty clearing sputum.

- What is the potential for adverse effects from the therapy? Which therapy is most likely to provide the greatest benefit with the least harm?

- What is the cost of the equipment for this therapy? The cost of the device may not be covered by insurance, resulting in considerable out-of-pocket expense for the patient or the hospital.

- What are the preferences of the patient? Lacking evidence that any technique is superior to another, patient preference is an important consideration.

When a clinical decision is made to try a secretion clearance technique, an $\mathrm{N}$ of 1 trial can be conducted.

\section{Aerosol Device}

There are advantages and disadvantages associated with each aerosol device category (nebulizer, metered-dose inhaler, dry powder inhaler). But the variety of devices has resulted in a confusing number of choices for clinicians who are selecting an aerosol delivery device for an individual patient. Dolovich and colleagues ${ }^{60}$ prepared evidence-based guidelines for the selection of an appropriate aerosol delivery device in specific clinical settings. A systematic review of pertinent RCTs was undertaken. Of the 131 studies that met the eligibility criteria, only 59 studies, primarily those that tested $\beta_{2}$ agonists, had useable data. None of the pooled meta-analyses showed a significant difference between devices in any efficacy outcome in any patient group for each of the clinical settings that was investigated. The authors concluded that aerosol delivery devices used for the delivery of bronchodilators and steroids can be equally efficacious. When selecting an aerosol delivery device for patients with asthma and COPD, they suggest that the following should be considered:

- Device/drug availability

- Clinical setting

- Patient age and the ability to use the selected device correctly

- Device use with multiple medications

- Cost and reimbursement

- Drug administration time

- Convenience in both out-patient and in-patient settings

- Physician and patient preference

The authors also point out the crucial importance of patient instruction in the proper use of the device.

\section{PEEP for ARDS}

A matter of controversy is how to set PEEP for the patient with ARDS. To date, 6 clinical trials have assessed the application of lower versus higher PEEP in patients with ARDS. ${ }^{61,62}$ Only 2 reported a significant mortality reduction with higher PEEP In these studies, however, 2 
interventions were applied: a higher PEEP was combined with a lower $\mathrm{V}_{\mathrm{T}}$. Thus, it is unknown whether the mortality benefit was related to the higher PEEP, lower $\mathrm{V}_{\mathrm{T}}$, or a combined effect. There are 5 meta-analyses published on the topic of higher versus lower PEEP in patients with ARDS. Of these, 4 found no effect on mortality from higher PEEP, compared with moderate PEEP.

The meta-analysis by Briel et $\mathrm{al}^{63}$ is unique in that data from 2,299 individual patients in 3 trials were analyzed, using uniform outcome definitions. The mortality rate was $33 \%$ for patients assigned to treatment with higher PEEP, and $35 \%$ for patients assigned to lower PEEP (adjusted relative risk $0.94,95 \% \mathrm{CI} 0.86-1.04, P=.25$ ). However, in patients with moderate to severe ARDS the mortality was 34\% in the higher-PEEP group and 39\% in the lowerPEEP group (adjusted relative risk 0.90, 95\% CI 0.81$1.00, P=.049)$. In patients with mild ARDS the mortality was $27 \%$ in the higher-PEEP group and $19 \%$ in the lowerPEEP group (adjusted relative risk 1.37, 95\% CI $0.98-$ $1.92, P=.07)$. The results of this meta-analysis suggest that treatment with higher PEEP was associated with improved survival among the subgroup of patients with moderate to severe ARDS. But there was a trend toward worse outcomes with higher PEEP in the subgroup with mild ARDS. There are many techniques that can be used to select PEEP, but evidence is lacking for superiority of one approach, compared to the others.

\section{Why Isn't the Best Evidence Implemented Into Practice?}

The tenets of EBM are not universally accepted. A variety of reasons have been given to support a practice not consistent with EBM (Table 7). Some clinicians do not accept a hierarchy of evidence, or grading of the evidence, arguing instead that experiential evidence and evidence from physiologic trials are equally important, or perhaps even more important, than evidence by well done, prospective randomized trials in humans. ${ }^{64-66}$ Clinical wisdom is required to determine whether or not the evidence is valid and how the evidence is best applied to the care of an individual patient. It is often pointed out that high-level evidence does not exist for many respiratory care practices. However, that is no excuse for not implementing high-level evidence when it is available. EBM does not discredit the value of observational studies, physiologic studies, and clinical experience. Such evidence is important to assess mechanisms of disease and to establish proof of principle. However, lower levels of evidence should impact clinical practice less than the results of a well done RCT.

It has been reported that about $30-40 \%$ of patients do not receive care according to best evidence. The ARDS Network study ${ }^{18}$ is a case in point. Despite the strong
Table 7. Common Reasons Why Clinicians Do Not Practice Evidence-Based Medicine

\footnotetext{
They do not recognize the evidence (laziness)

They do not read the literature

They do not attend conferences

They do not talk to their colleagues

They do not believe the evidence (ignorance)

They do not believe the right study was done (eg, wrong dose)

The study is not consistent with lower levels of evidence (eg, animal studies)

They believe that incorporating the evidence into practice is someone else's job (blaming)

The system prevents incorporating the latest evidence

There is not enough time

The right resources are not available

There is not hospital policy supporting the practice change

They do not think the evidence applies to their practice (stubbornness)

Their patients are sicker

Their patients are older

Their patients have always done fine with their "expert" treatment
}

(From reference 4.)

finding that mortality is lower with a $\mathrm{V}_{\mathrm{T}}$ of $6 \mathrm{~mL} / \mathrm{kg}$, compared to $12 \mathrm{~mL} / \mathrm{kg}$, the practice of using lower $\mathrm{V}_{\mathrm{T}}$ in patients with ARDS remains under-utilized. In 13 ICUs at 4 hospitals in Baltimore, Maryland, only $41 \%$ of eligible ventilator settings collected from 2004 to 2007 were adherent to lung-protective ventilation, and $37 \%$ of patients never received lung-protective ventilation. Another case in point is NIV, where it has been reported in several studies that this therapy is under-utilized. ${ }^{67-69}$ In 1 study of patients admitted to ICUs with a diagnosis of either COPD or congestive heart failure exacerbation, only a third had a trial of NIV.70

\section{Summary}

EBM has permeated all parts of healthcare practice, including respiratory care. The principles of EBM provide us the tools to incorporate the best evidence into our everyday practice. Although all tenets of EBM are not universally accepted, the principles of EBM nonetheless provide a valuable approach to improve respiratory care practice.

\section{REFERENCES}

1. Pierce AK, Saltzman HA. Conference on the scientific basis of respiratory therapy. Am Rev Respir Dis 1974;110(6 Pt 2):1-3.

2. Evidence-Based Medicine Working Group. Evidence-based medicine. A new approach to teaching the practice of medicine. JAMA 1992;268(17):2420-2425.

3. Guyatt G, Rennie D, Meade M, Cook D. Users' guides to the medical literature. Essentials of evidence-based practice. New York: McGraw Hill; 2008. 


\section{Science and Evidence: Separating Fact from Fiction}

4. Hess DR. What is evidence-based medicine and why should I care? Respir Care 2004;49(7):730-741.

5. Sackett DL, Straus SE, Richardson WS, Rosenberg W, Haynes RB. Evidence-based medicine. How to practice and teach EBM. New York: Churchill Livingstone; 2000.

6. Sackett DL. Clinician-trialist rounds. 4. Why not do an n-of-1 RCT? Clin Trials 2011;8(3):350-352.

7. Lillie EO, Patay B, Diamant J, Issell B, Topol EJ, Schork NJ. The n-of-1 clinical trial: the ultimate strategy for individualizing medicine? Per Med 2011;8(2):161-173.

8. Gabler NB, Duan N, Vohra S, Kravitz RL. N-of-1 trials in the medical literature: a systematic review. Med Care 2011;49(8):761-768.

9. Wheeler DM. High-frequency chest-wall compression, patient safety, and the n-of-1 construct. Respir Care 2009;54(3):322-323.

10. Hess DR. Airway clearance: physiology, pharmacology, techniques, and practice. Respir Care 2007;52(10):1392-1396.

11. Nikles CJ, Clavarino AM, Del Mar CB. Using n-of-1 trials as a clinical tool to improve prescribing. Brit J Gen Pract 2005;55(512): 175-180.

12. Madhok V, Fahey T. N-of-1 trials: an opportunity to tailor treatment in individual patients. Brit J Gen Pract 2005;55(512):171-172.

13. Mahon JL, Laupacis A, Hodder RV, McKim DA, Paterson NA, Wood TE, et al. Theophylline for irreversible chronic airflow limitation: a randomized study comparing $\mathrm{n}$ of 1 trials to standard practice. Chest 1999;115(1):38-48.

14. Senn S. Applying results of randomised trials to patients. $\mathrm{N}$ of 1 trials are needed. BMJ 1998;317(7157):537-538.

15. Mahon J, Laupacis A, Donner A, Wood T. Randomized study of $n$ of 1 trials versus standard practice. BMJ 1996;312(7038):1069-1074.

16. Patel A, Jaeschke R, Guyatt GH, Keller JL, Newhouse MT. Clinical usefulness of n-of-1 randomized controlled trials in patients with nonreversible chronic airflow limitation. Am Rev Respir Dis 1991; 144(4):962-964.

17. Smith GC, Pell JP. Parachute use to prevent death and major trauma related to gravitational challenge: systematic review of randomised controlled trials. BMJ 2003;327(7429):1459-1461.

18. Acute Respiratory Distress Syndrome Network. Ventilation with lower tidal volumes as compared with traditional tidal volumes for acute lung injury and the acute respiratory distress syndrome. N Engl J Med 2000;342(18):1301-1308.

19. Dellinger RP, Zimmerman JL, Taylor RW, Straube RC, Hauser DL, Criner GJ, et al; Inhaled Nitric Oxide in ARDS Study Group. Effects of inhaled nitric oxide in patients with acute respiratory distress syndrome: results of a randomized phase II trial. Crit Care Med 1998;26(1):15-23.

20. Delclaux C, L'Her E, Alberti C, Mancebo J, Abroug F, Conti G, et al. Treatment of acute hypoxemic nonhypercapnic respiratory insufficiency with continuous positive airway pressure delivered by a face mask: a randomized controlled trial. JAMA 2000;284(18): 2352-2360.

21. Wilton NK, Slim AM. Application of the principles of evidencebased medicine to patient care. South Med J 2012;105(3):136-143.

22. Hess DR. Information retrieval in respiratory care: tips to locate what you need to know. Respir Care 2004;49(4):389-399; discussion 399-400.

23. Chatburn RL. How to find the best evidence. Respir Care 2009; 54(10):1360-1365.

24. Citrome L, Moss SV, Graf C. How to search and harvest the medical literature: let the citations come to you, and how to proceed when they do. Int J Clin Prac 2009;63(11):1565-1570.

25. Callcut RA, Branson RD. How to read a review paper. Respir Care 2009;54(10):1379-1385.
26. Murad MH, Montori VM. Synthesizing evidence: shifting the focus from individual studies to the body of evidence. JAMA 2013;309(21): 2217-2218.

27. Hess DR. Evidence-based clinical practice guidelines: where's the evidence and what do I do with it? Respir Care 2003;48(9):838-839.

28. Restrepo RD. AARC Clinical Practice Guidelines: from "referencebased" to "evidence-based". Respir Care 2010;55(6):787-789.

29. Brozek JL, Akl EA, Alonso-Coello P, Lang D, Jaeschke R, Williams $\mathrm{JW}$, et al. Grading quality of evidence and strength of recommendations in clinical practice guidelines. Part 1 of 3 . An overview of the GRADE approach and grading quality of evidence about interventions. Allergy 2009;64(5):669-677.

30. Brozek JL, Akl EA, Compalati E, Kreis J, Terracciano L, Fiocchi A, et al. Grading quality of evidence and strength of recommendations in clinical practice guidelines part 3 of 3 . The GRADE approach to developing recommendations. Allergy 2011;66(5):588-595.

31. Brozek JL, Akl EA, Jaeschke R, Lang DM, Bossuyt P, Glasziou P, et al. Grading quality of evidence and strength of recommendations in clinical practice guidelines: Part 2 of 3 . The GRADE approach to grading quality of evidence about diagnostic tests and strategies. Allergy 2009;64(8):1109-1116.

32. Guyatt G, Gutterman D, Baumann MH, Addrizzo-Harris D, Hylek EM, Phillips B, et al. Grading strength of recommendations and quality of evidence in clinical guidelines: report from an American College of Chest Physicians Task Force. Chest 2006;129(1):174-181.

33. Guyatt GH, Oxman AD, Kunz R, Jaeschke R, Helfand M, Liberati A, et al. Incorporating considerations of resources use into grading recommendations. BMJ 2008;336(7654):1170-1173.

34. Guyatt GH, Oxman AD, Kunz R, Vist GE, Falck-Ytter Y, Schünemann HJ, et al. What is "quality of evidence" and why is it important to clinicians? BMJ 2008;336(7651):995-998.

35. Guyatt GH, Oxman AD, Vist GE, Kunz R, Falck-Ytter Y, AlonsoCoello P, et al. GRADE: an emerging consensus on rating quality of evidence and strength of recommendations. BMJ 2008;336(7650): 924-926.

36. Jaeschke R, Guyatt GH, Dellinger P, Schünemann H, Levy MM, Kunz R, et al. Use of GRADE grid to reach decisions on clinical practice guidelines when consensus is elusive. BMJ 2008;337:a744.

37. Kavanagh BP. The GRADE system for rating clinical guidelines. PLoS Med 2009;6(9):e1000094.

38. Schünemann HJ, Oxman AD, Brozek J, Glasziou P, Bossuyt P, Chang S, et al. GRADE: assessing the quality of evidence for diagnostic recommendations. ACP J Club 2008;149(6):2.

39. Ransohoff DF, Pignone M, Sox HC. How to decide whether a clinical practice guideline is trustworthy. JAMA 2013;309(2):139-140.

40. Ram FS, Lightowler JV, Wedzicha JA. Non-invasive positive pressure ventilation for treatment of respiratory failure due to exacerbations of chronic obstructive pulmonary disease. Cochrane Database Syst Rev 2003;(1):CD004104.

41. Vital FM, Saconato H, Ladeira MT, Sen A, Hawkes CA, Soares B, et al. Non-invasive positive pressure ventilation (CPAP or bilevel NPPV) for cardiogenic pulmonary edema. Cochrane Database Syst Rev 2008(3):CD005351.

42. Keenan SP, Sinuff T, Burns KE, Muscedere J, Kutsogiannis J, Mehta S, et al. Clinical practice guidelines for the use of noninvasive positive-pressure ventilation and noninvasive continuous positive airway pressure in the acute care setting. CMAJ 2011;183(3): E195-E214.

43. Williams JW Jr, Cox CE, Hargett CW, Gilstrap DL, Castillo CE, Govert JA, et al. Noninvasive positive-pressure ventilation (NPPV) for acute respiratory failure. Comparative effectiveness reviews no. 68. Rockville, MD: Agency for Healthcare Research and Quality; 2012. Report No. 12-EHC089-EF. 
44. Needham DM, Colantuoni E, Mendez-Tellez PA, Dinglas VD, Sevransky JE, Dennison Himmelfarb CR, et al. Lung protective mechanical ventilation and two year survival in patients with acute lung injury: prospective cohort study. BMJ 2012;344:e2124.

45. Serpa Neto A, Cardoso SO, Manetta JA, Pereira VG, Esposito DC, Pasqualucci Mde $\mathrm{O}$, et al. Association between use of lungprotective ventilation with lower tidal volumes and clinical outcomes among patients without acute respiratory distress syndrome: a metaanalysis. JAMA 2012;308(16):1651-1659.

46. MacIntyre NR, Cook DJ, Ely EW Jr, Epstein SK, Fink JB, Heffner $\mathrm{JE}$, et al. Evidence-based guidelines for weaning and discontinuing ventilatory support: a collective task force facilitated by the American College of Chest Physicians; the American Association for Respiratory Care; and the American College of Critical Care Medicine. Chest 2001;120(6 Suppl):375S-395S.

47. Blackwood B, Alderdice F, Burns K, Cardwell C, Lavery G, O'Halloran P. Use of weaning protocols for reducing duration of mechanical ventilation in critically ill adult patients: Cochrane systematic review and meta-analysis. BMJ 2011;342:c7237.

48. Meade M, Guyatt G, Cook D, Griffith L, Sinuff T, Kergl C, et al. Predicting success in weaning from mechanical ventilation. Chest 2001;120(6 Suppl):400S-424S.

49. Tanios MA, Nevins ML, Hendra KP, Cardinal P, Allan JE, Naumova $\mathrm{EN}$, et al. A randomized, controlled trial of the role of weaning predictors in clinical decision making. Crit Care Med 2006;34(10): 2530-2535.

50. National Heart, Lung, and Blood Institute Acute Respiratory Distress Syndrome (ARDS) Clinical Trials Network; Matthay MA, Brower RG, Carson S, Douglas IS, et al. Randomized, placebo-controlled clinical trial of an aerosolized $\beta_{2}$-agonist for treatment of acute lung injury. Am J Respir Crit Care Med 2011;184(5):561-568.

51. Gao Smith F, Perkins GD, Gates S, Young D, McAuley DF, Tunnicliffe $\mathrm{W}$, et al. Effect of intravenous $\beta$-2 agonist treatment on clinical outcomes in acute respiratory distress syndrome (BALTI-2): a multicentre, randomised controlled trial. Lancet 2012;379(9812): 229-235.

52. Fessler HE, Hess DR. Respiratory controversies in the critical care setting. Does high-frequency ventilation offer benefits over conventional ventilation in adult patients with acute respiratory distress syndrome? Respir Care 2007;52(5):595-605; discussion 606-608.

53. Sud S, Sud M, Friedrich JO, Meade MO, Ferguson ND, Wunsch H, et al. High frequency oscillation in patients with acute lung injury and acute respiratory distress syndrome (ARDS): systematic review and meta-analysis. BMJ 2010;340:c2327.

54. Ferguson ND, Cook DJ, Guyatt GH, Mehta S, Hand L, Austin P, et al. High-frequency oscillation in early acute respiratory distress syndrome. N Engl J Med 2013;368(9):795-805.

55. Young D, Lamb SE, Shah S, MacKenzie I, Tunnicliffe W, Lall R, et al. High-frequency oscillation for acute respiratory distress syndrome. N Engl J Med 2013;368(9):806-813.
56. Bollen CW, van Well GT, Sherry T, Beale RJ, Shah S, Findlay G, et al. High frequency oscillatory ventilation compared with conventional mechanical ventilation in adult respiratory distress syndrome: a randomized controlled trial [ISRCTN24242669]. Crit Care 2005; 9(4):R430-R439.

57. Derdak S, Mehta S, Stewart TE, Smith T, Rogers M, Buchman TG, et al. High-frequency oscillatory ventilation for acute respiratory distress syndrome in adults: a randomized, controlled trial. Am J Respir Crit Care Med 2002;166(6):801-808.

58. Flume PA, Robinson KA, O'Sullivan BP, Finder JD, Vender RL, Willey-Courand DB, et al; Clinical Practice Guidelines for Pulmonary Therapies Committee. Cystic fibrosis pulmonary guidelines: airway clearance therapies. Respir Care 2009;54(4):522-537.

59. Ides K, Vissers D, De Backer L, Leemans G, De Backer W. Airway clearance in COPD: need for a breath of fresh air? A systematic review. COPD 2011;8(3):196-205.

60. Dolovich MB, Ahrens RC, Hess DR, Anderson P, Dhand R, Rau JL, et al; American College of Chest Physicians; American College of Asthma, Allergy, and Immunology. Device selection and outcomes of aerosol therapy: evidence-based guidelines. Chest 2005;127(1): 335-371.

61. Hess DR. Approaches to conventional mechanical ventilation of the patient with acute respiratory distress syndrome. Respir Care 2011; 56(10):1555-1572.

62. Hess DR. How much PEEP? Do we need another meta-analysis? Respir Care 2011;56(5):710-713.

63. Briel M, Meade M, Mercat A, Brower RG, Talmor D, Walter SD, et al. Higher vs lower positive end-expiratory pressure in patients with acute lung injury and acute respiratory distress syndrome: systematic review and meta-analysis. JAMA 2010;303(9):865-873.

64. Tobin MJ. Counterpoint: evidence-based medicine lacks a sound scientific base. Chest 2008;133(5):1071-1074; discussion 1074-1077.

65. Tobin MJ. The role of a journal in a scientific controversy. Am J Respir Crit Care Med 2003;168(5):511.

66. Tobin MJ. Culmination of an era in research on the acute respiratory distress syndrome. N Engl J Med 2000;342(18):1360-1361.

67. Maheshwari V, Paioli D, Rothaar R, Hill NS. Utilization of noninvasive ventilation in acute care hospitals: a regional survey. Chest 2006;129(5):1226-1233.

68. Hess DR, Pang JM, Camargo CA Jr. A survey of the use of noninvasive ventilation in academic emergency departments in the United States. Respir Care 2009;54(10):1306-1312.

69. Bierer GB, Soo Hoo GW. Noninvasive ventilation for acute respiratory failure: a national survey of Veterans Affairs hospitals. Respir Care 2009;54(10):1313-1320.

70. Sweet DD, Naismith A, Keenan SP, Sinuff T, Dodek PM. Missed opportunities for noninvasive positive pressure ventilation: a utilization review. J Crit Care 2008;23(1):111-117. 